\title{
Step Patterns on Vicinal Reconstructed Surfaces
}

\author{
Igor Vilfan \\ J. Stefan Institute, P.O. Box 100, SI-61111 Ljubljana, Slovenia \\ e-mail: igor.vilfan@ijs.si
}

\begin{abstract}
Step patterns on vicinal $(2 \times 1)$ reconstructed surfaces of noble metals $\mathrm{Au}(110)$ and $\mathrm{Pt}(110)$, miscut towards the (100) orientation, are investigated. The free energy of the reconstructed surface with a network of crossing opposite steps is calculated in the strong chirality regime when the steps cannot make overhangs. It is explained why the steps are not perpendicular to the direction of the miscut but form in equilibrium a network of crossing steps which make the surface to look like a fish skin. The network formation is the consequence of competition between the - predominantly elastic - energy loss and entropy gain. It is in agreement with recent scanning-tunnelling-microscopy observations on vicinal $\mathrm{Au}(110)$ and $\mathrm{Pt}(110)$ surfaces.
\end{abstract}

Key words: Vicinal single-crystal surfaces. Surface structure, morphology, roughness, and topography. Surface relaxation and reconstruction. Surface thermodynamics. Gold, platinum.

\section{Introduction}

Vicinal surfaces inevitably have steps which are, in general, perpendicular to the miscut direction. For the $(2 \times 1)$ reconstructed surfaces like $\mathrm{Au}(110)$ and $\operatorname{Pt}(110)$, however, the situation is different when the surface is miscut towards the (010) orientation. Recent scanning-tunnelling microscopy (STM) observations revealed that the single-height difference steps are not parallel to $[00 \overline{1}]$, but run roughly in the $[1 \overline{1} 1]$ and $[1 \overline{1} \overline{1}]$ directions $[1,2]$. The steps thus cross and form a network, also called "textured pattern" or "fish skin," according to the specific shape of the terraces bound by the steps.

A specific property of the steps on reconstructed FCC (110) surfaces is the anisotropy in their energy. The step energy is high (of the order $0.1 \mathrm{eV}$ ) for 


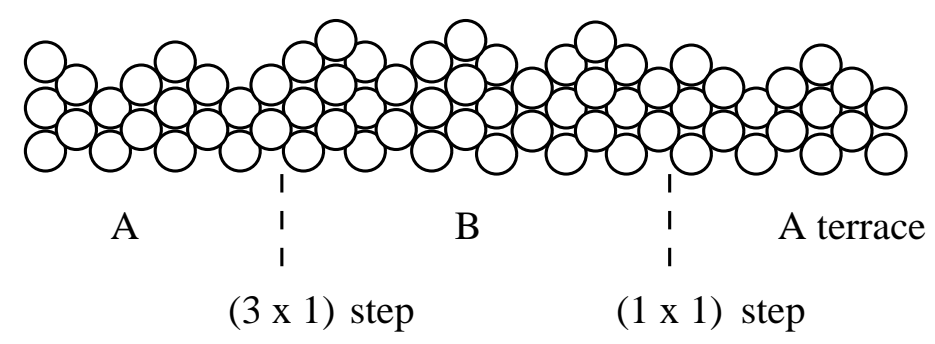

(a)

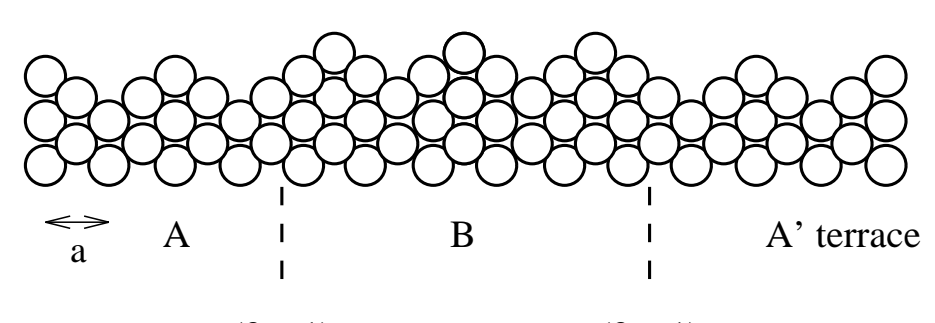

$(3 \times 1)$ step

(3 x 1) step

(b)

Fig. 1. Cross section through the $(2 \times 1)$ reconstructed $(110)$ surface of an FCC crystal. (a) A $(3 \times 1)$ and a $(1 \times 1)$ step. (b) Two opposite $(3 \times 1)$ steps.

steps perpendicular to the missing rows and low $\left(\sim 10^{-3} \mathrm{eV}\right)$ when they are parallel to the missing rows. Two types of low-energy single-height-difference steps can be formed, the $(3 \times 1)$ and the $(1 \times 1)$ steps, after the size of their exposed (111) facets, see Fig. 1. The two types of steps have opposite lateral shifts in the topmost atomic row positions so that the rows on the right hand and on the left hand terraces are in phase. In general, the two types of steps have different energies. Calculation with the embedded-atom method tells us that the $(3 \times 1)$ steps have lower energy than the $(1 \times 1)$ steps [3]. Indeed, the $(3 \times 1)$ are also the predominant steps seen in STM on $\mathrm{Au}(110)$ and $\mathrm{Pt}(110)$ $[1,2]$. The difference in energy is connected with chirality, i.e., the $A-B$ and the $B-A$ steps are not equivalent [4]. In the strong chirality regime when the energy of the $(1 \times 1)$ step is much higher than the energy of the $(3 \times 1)$ step, only the $(3 \times 1)$ steps are thermally excited. Here we will consider the strong chirality case so that the presence of the $(1 \times 1)$ steps is negligible. At each $(3 \times 1)$ step the phase in the position of the topmost atomic rows is shifted laterally by $+\pi / 2$. Two $(3 \times 1)$ steps together thus cause a phase shift of $\pi$, they form an Ising (antiphase) domain boundary, the missing rows on the terraces $A$ and $A^{\prime}$ are out of phase, see Fig. 1(b). For this reason, the $(3 \times 1)$ steps cannot make overhangs (loops back, shown in Fig. $2(\mathrm{a}))$ in the 


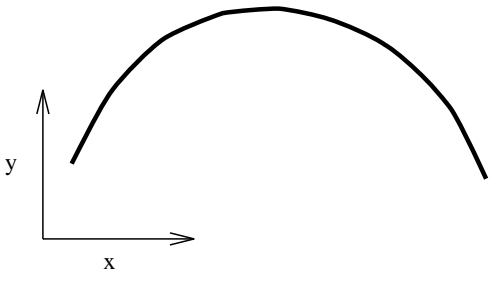

(a)

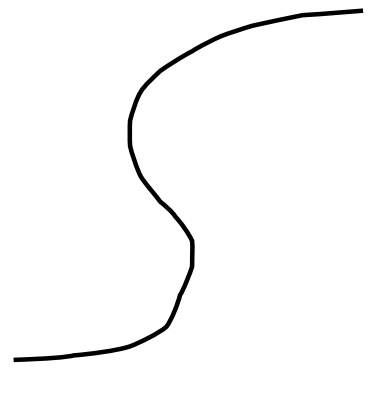

(b)

Fig. 2. Overhangs of a step on a reconstructed surface. (a) Because of the $(2 \times 1)$ reconstruction, the overhangs in the $y$ direction of a $(3 \times 1)$ step alone are not allowed, only overhangs where the $(3 \times 1)$ step changes into a $(1 \times 1)$ are possible. (b) The overhangs in the $x$ direction increase the length, projected on the $x$ axis and thus increase the number of broken atomic bonds.

[110] direction [5]. If the overhangs were allowed, then one can get from $A$ to $A^{\prime}$ around the overhang without crossing any domain boundary or step, so the $A$ and $A^{\prime}$ terraces should be in phase. Like in the four-state Potts model, (a multiple of) four steps must meet in a vertex (at least two lines cross or four lines terminate).

As already mentioned, a miscut of a (110) surface towards the (010) orientation is expected to produce steps, parallel to the [001] $(x)$ axis. The energy of such steps is very high because some additional surface-atoms bonds are broken there. Meandering of steps in the $y([\overline{1} 10])$ direction, on the other hand, creates sections, parallel to the $y$ direction, which have much lower energy than the sections parallel to the $x$ axis. At finite temperature, therefore, the steps are not straight lines along the $x$ axis but make many thermally excited excursions in the $y$ direction. However, as the overhangs in the $y$ direction are forbidden in the strong chirality regime, and the overhangs in the $x$ direction are energetically costly and rare as they increase the number of broken bonds (see Fig. 2(b)), the step excursions are possible only in one direction. Depending on the orientation of steps, the step excursions are either in the $+y$ ("up," left-hand step in Fig. 1(b)) or in the $-y$ direction ("down," right-hand step in Fig. 1(b)). Notice that the steps of the same kind cannot cross. When the imposed inclination is exactly in the $y$ direction, there must be an equal density of "up" and "down" steps which inevitably interpenetrate, the steps form a network, shown schematically in Fig. 3, which was also observed in the scanning-tunnelling microscopy $[1,2]$.

The aim of this paper is to investigate the stability of step patterns. Special emphasis will be on thermally-induced but chirality-restricted meandering of steps and on the elastic interactions between them. In the following Section we shall analyse the energy and entropy of different step configurations and of the step crossings. The elastic interaction energy between the steps, calculated in 


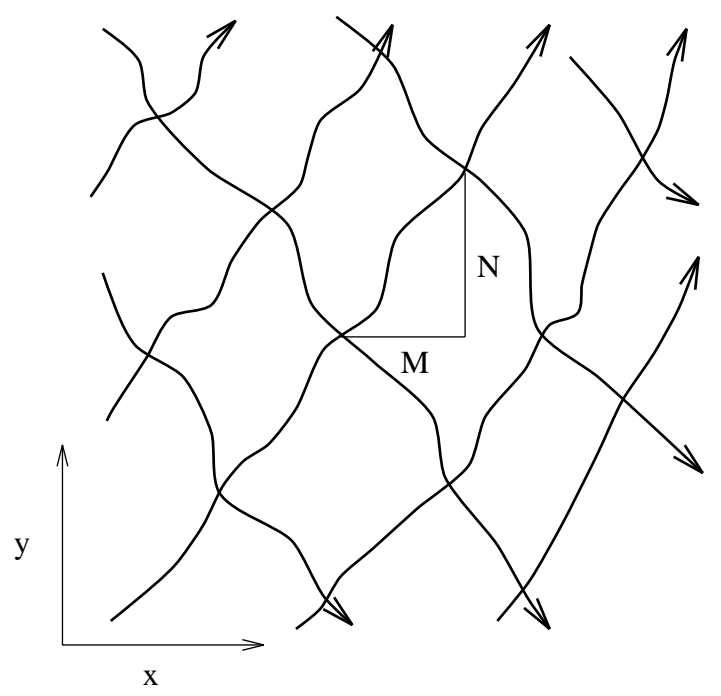

Fig. 3. View on a vicinal reconstructed (110) surface, miscut towards (100). The missing rows are parallel to the $y$ axis. The $(3 \times 1)$ steps are either "up" or "down" and form a characteristic pattern. Terraces between the steps resemble on a fish skin. The steps make no overhangs and the steps of the same kind never cross. The average separations between the step crossings, vertices, are $M$ and $N$ in the $x$ and $y$ directions, respectively.

Section 3, will be used to evaluate the free energy in Section 4. In Section 5 the model will be applied to the reconstructed surfaces of $\mathrm{Au}(110)$ and $\operatorname{Pt}(110)$.

\section{Step Energetics}

At a single-height step which is perpendicular to the missing rows, one atomic bond along the missing rows is broken per unit length $a$. The step energy $V$ (per unit length) is high $\left(V \gg k_{B} T\right)$, a step is seldomly thermally excited in this direction. However, with the miscut towards the (010) orientation, such steps are imposed on the surface. The average step separation in the $y$ direction (measured in units of atom spacings $a / \sqrt{2})$ is $N+1 / 2=(2 \tan \theta)^{-1}$ where $N$ is the average number of atoms in a top row on a terrace and $\theta$ the miscut angle. Meandering without overhangs introduces sections of steps parallel to the $y$ direction but maintains the total number of broken bonds constant. The energy of such sections (per $a / \sqrt{2}$ ) is denoted by $\epsilon$ and is much smaller than $V$. If we neglect the energies of kinks and take into account that the overhangs are forbidden by the strong chirality condition, the energy of a step that extends $M$ unit cells in the $x$ and $N$ atom spacings in the $y$ directions (see Fig. 3) is simply $M V+N \epsilon$.

Meandering of steps introduces also vertices, crossings of "up" with "down" steps. At a vertex two opposite steps meet and form a short segment (length 
$\left.N^{\prime} \ll N\right)$ of a narrow Ising domain wall oriented parallel to the $y$ axis and with the energy $\eta N^{\prime}$ [6]. The vertex energy is $W=(\eta-2 \epsilon) N^{\prime}$ and it is related to the short-range elastic interaction between the steps. From the experimental evidence of separate Ising (deconstruction) and roughening (or wetting) transitions $[7,8]$, the conclusion was reached that the interaction is weak but attractive, i.e., $\eta<2 \epsilon$. The average density of vertices is $(N M)^{-1}$. $N$ is determined by the miscut angle and $M$ will be a variational parameter, related to the average angle $\phi$ at which two opposite steps cross: $\tan (\phi / 2)=$ $\sqrt{2} M / N$.

Thermally induced step fluctuations increase the step energy on one hand but also the entropy on the other hand. In the following, we shall approximate the step texture by a regular lattice of vertices connected by step segments which fluctuate without overhangs. A step segment with fixed ends at $(0,0)$ and $(M, N)$ has

$$
P=\frac{(M+N) !}{M ! N !}
$$

different configurations with the same step energy. The free energy of such a segment and one vertex is

$$
F(M, N)=M V+N \epsilon-k_{B} T \ln \frac{(M+N) !}{M ! N !}+W .
$$

This free energy expression is minimal at finite $M / N$. However, this step free energy is not complete and the minimum is at wrong $M / N$. In the following Section the elastic contribution to the free energy will be calculated.

\section{Elastic Interaction Between Steps}

Long-range elastic interaction between steps has been first discussed by Marchenko and Parshin [9]; see also [10-12]. A step exerts elastic forces which result in an elastic displacement field in the bulk. The displacement field of a step interacts with the elastic forces of other steps and the interaction energy is the elastic contribution to the surface free energy density.

In the continuum elasticity theory, the force on the underlying elastic continuum exerted by the step $i$ running parallel to the $y^{\prime}$ axis (which is, in general, not parallel to $y$ ) is described by a force doublet localized at the step $i[9-11]$ :

$$
\vec{F}_{i}\left(x^{\prime}\right)=\vec{f} \delta^{\prime}\left(x^{\prime}\right) .
$$


$f_{x^{\prime}}=f_{\|}$describes the local stretch of the surface which is the same for the "up" and "down" steps, and $f_{z}=\tau a / \sqrt{8}$ is a local torque, which tends to twist the crystal around the $y^{\prime}$ axis, $\tau$ is the (tangential) surface stress.

As usually, we will calculate the elastic energy neglecting the fluctuations of the step (assuming straight lines) and assuming an isotropic bulk.

\subsection{Elastic energy of parallel steps}

The elastic interaction energy between two parallel steps $i$ and $j$ a distance $\ell$ apart is [9-11]:

$$
E_{i j}=\frac{2\left(1-\sigma^{2}\right)}{\pi E} \frac{\vec{f}_{1} \cdot \vec{f}_{2}}{\ell^{2}}=\frac{2\left(1-\sigma^{2}\right)}{\pi E} \frac{f_{\|}^{2}+f_{z}^{2}}{\ell^{2}},
$$

where $E$ is the Young modulus and $\sigma$ the Poisson ratio. When the surface is covered with an infinite array of equidistant parallel steps, the step interacts with all the other steps. If $\ell$ is the distance between two neighbouring steps, the elastic contribution to the surface energy density is

$$
e_{p}=\frac{1}{\ell} \sum_{i>j} E_{i j}=\frac{\pi\left(1-\sigma^{2}\right)}{3 E} \frac{f_{\|}^{2}+f_{z}^{2}}{\ell^{3}},
$$

parallel steps of the same kind always repel.

\subsection{Elastic energy of two crossing steps}

Now we will calculate the elastic interaction energy of an "up" step with a "down" step that cross at $\left(x^{\prime}, y^{\prime}\right)=0$. Let the step 1 be along the $y^{\prime}$ axis and let the angle between the steps be $\phi$. The elastic interaction energy, if both steps are infinitely long, is:

$$
E_{12}(\phi)=-\int_{-\infty}^{+\infty} \mathrm{dx}^{\prime} \mathrm{dy}^{\prime} \overrightarrow{\mathrm{F}}_{1}\left(\mathrm{x}^{\prime}\right) \cdot \overrightarrow{\mathrm{u}}_{2}\left(\mathrm{x}^{\prime}, \mathrm{y}^{\prime}\right),
$$

where $\vec{u}$ is the displacement field of the second step at $\left(x^{\prime}, y^{\prime}\right)$,

$$
\vec{u}_{2}=-\frac{2\left(1-\sigma^{2}\right)}{\pi E} \frac{\vec{f}_{2}}{r}
$$


and $r$ the shortest distance between the point $\left(x^{\prime}, y^{\prime}\right)$ and the step 2,

$$
r=\left|x^{\prime} \cos \phi+y^{\prime} \sin \phi\right|
$$

Since the two crossing steps are always opposite to one another, we have $f_{2, z}=-f_{1, z}$, whereas $f_{2, x^{\prime}}=f_{\|} \cos \phi$. Partial integration of (6) over $x^{\prime}$ yields:

$$
E_{12}(\phi)=\frac{2\left(1-\sigma^{2}\right)}{\pi E} \int_{-\infty}^{+\infty} \frac{\mathrm{dy}^{\prime}}{\mathrm{r}^{2}}\left[f_{\|}^{2} \cos ^{2} \phi+f_{z}^{2} \cos \phi\right]
$$

Obviously, the continuum theory breaks down for small $r$, therefore we integrate over $|r|>a$ and incorporate the remaining short-range interaction into the vertex energy $W$. The energy of two crossing steps is then:

$$
E_{12}(\phi)=\frac{4\left(1-\sigma^{2}\right)}{\pi E a}\left[f_{\|}^{2} \cos ^{2} \phi-f_{z}^{2} \cos \phi\right] \frac{1}{\sin \phi}+W .
$$

The expression (10) diverges for $\phi=0$ when the steps overlap. Nevertheless, we notice that the square bracket reduces to $f_{\|}^{2}+f_{z}^{2}$ for parallel steps of the same kind $(\phi \rightarrow \pi)$ and to $f_{\|}^{2}-f_{z}^{2}$ for opposite (antiparallel, $\phi \rightarrow 0$ ) steps, in agreement with [9]. From Eq. (10) it is also evident that two perpendicular steps do not interact elastically.

\section{The free energy density}

Using the results of the previous Sections, the free energy density (per unreconstructed surface unit cell) becomes equal to:

$$
\begin{array}{r}
f(\xi)=a+\frac{b}{\xi}+\frac{\left(c-d \xi^{2}\right)\left(1-2 \xi^{2}\right)}{\xi^{2}\left(1+2 \xi^{2}\right)}+e \frac{\left(1+2 \xi^{2}\right)^{3 / 2}}{\xi^{3}} \\
-\frac{k_{B} T}{N}\left[\ln \left(\frac{1+\xi}{\xi}\right)+\frac{\ln (1+\xi)}{\xi}\right]
\end{array}
$$

where

$$
\begin{aligned}
& \xi=\frac{M}{N} \\
& a=\frac{V}{N},
\end{aligned}
$$




$$
\begin{gathered}
b=\frac{1}{N}\left(\epsilon+\frac{W}{N}\right), \\
c=\frac{\sqrt{2}\left(1-\sigma^{2}\right)}{\pi E N^{2} a}\left(f_{\|}^{2}-f_{z}^{2}\right), \\
d=\frac{2 \sqrt{2}\left(1-\sigma^{2}\right)}{\pi E N^{2} a}\left(f_{\|}^{2}+f_{z}^{2}\right), \\
e=\frac{\pi \sqrt{2}\left(1-\sigma^{2}\right)}{24 E N^{3} a}\left(f_{\|}^{2}+f_{z}^{2}\right)=\frac{\pi^{2}}{48 N} d .
\end{gathered}
$$

The first term in (11) is the energy of a step, parallel to the $x$ axis. At constant miscut angle, the first term does not influence the equilibrium shape of the step patterns, it only shifts the energy. The second term is the contribution of the step segments that are parallel to the $y$ axis and a contribution from the vertex energy. The third and the fourth terms are the elastic energies of crossing and parallel steps, respectively. The last term is the entropy contribution to the free energy assuming a regular lattice of vertices. The entropy tends to increase the density of steps and to decrease the angle between the opposite steps. Since the entropy is calculated for a step fluctuating only within a finite, $M \times N$ rectangle, the "loss of entropy" which leads to a $\sim 1 / \ell^{2}$ repulsion between parallel steps [10] is properly taken into account in (11).

In principle, the ratio $\xi=M / N$ is arbitrary, $0<\xi<\infty$. However, the free energy diverges for $\xi \ll 1$ and goes to $(d+2 \sqrt{2} e)$ for $\xi \gg 1$. $\xi$ is determined from the condition that $f$ is minimal and it is finite at finite temperature.

\section{The case of $\mathrm{Au}(110)$ and $\mathrm{Pt}(110)$}

The above results will now be applied to investigate the stability of step patterns on $\mathrm{Au}(110)$ and $\mathrm{Pt}(110)$ surfaces at room temperature $\left(k_{B} T=25 \mathrm{meV}\right)$. At this temperature, the surface diffusion (of $\mathrm{Au}$ ) is probably high enough to enable equilibration of steps on lengthscales of $\approx 100 \AA$ in less than an hour or so [13]. The parameters, used in the estimates are collected in Table 1. Since $f_{\|}$is not known, we take $\left|f_{\|} / f_{z}\right|=r$ as a parameter. The vertex energy is small $(W / N \ll \epsilon)$, therefore it will be neglected in calculating the free energy $(W=0)$.

Fig. 4 shows individual contributions to the free energy density (11) for vicinal $\mathrm{Au}(110)$ surfaces with $W=0$ and $r=4$. The free energy density is minimal at $\xi \approx 4$. At large $\xi$ (when the steps are almost parallel to the $x$ axis), the entropy term prevails whereas at small $\xi$ (when very dense steps are almost 


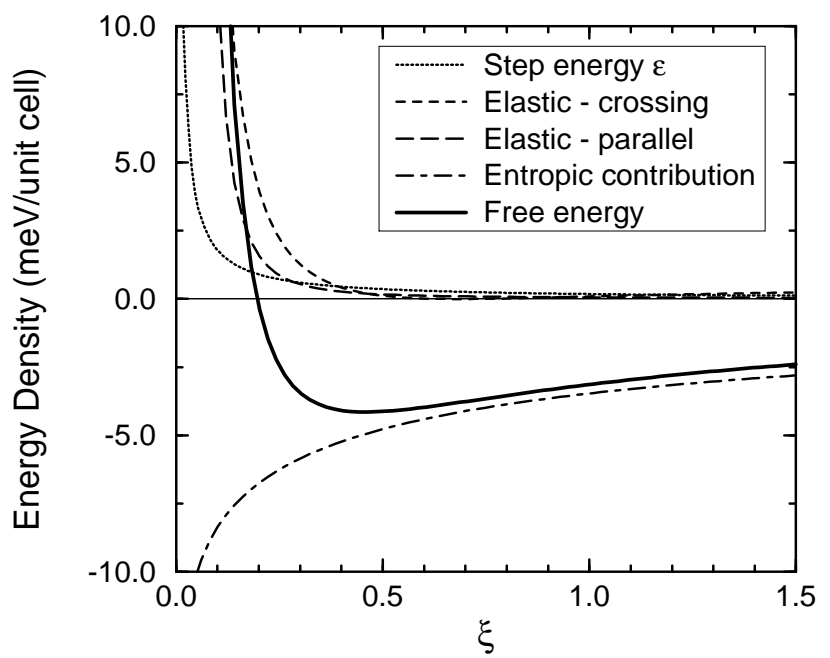

Fig. 4. Free energy density $f$ and individual contributions to $f$ for $\mathrm{Au}(110)$ surface with $2.7^{\circ}$ miscut $(N=10), W=0$ and $r=4$. The free energy density has a minimum at $\xi=M / N \approx 0.4$.

perpendicular to the $x$ axis) the energy density prevails. At short $\xi$, the elastic interaction energy is more important than the step energy. For $r>\sim 2$, the dominant contribution to the energy density comes from elastic interactions between crossing opposite steps.

The equilibrium values of the average angle $\phi$ between opposite steps as a function of the miscut angle $\theta$ for reconstructed Au and Pt (110) surfaces are shown in Fig. 5. Even for vanishingly small miscut angles the steps form the specific patterns. In the limit as $\theta \rightarrow 0$, when the step separation diverges $(N \rightarrow \infty)$, the elastic energy contributions per surface unit cell vanish as $1 / N^{2}$ or as $1 / N^{3}$, and the equilibrium step-crossing angle is given by

$$
\phi=2 \tan ^{-1}\left[\sqrt{2}\left(\mathrm{e}^{\epsilon / \mathrm{k}_{\mathrm{B}} \mathrm{T}}-1\right)\right] .
$$

Table 1

Parameters of the model for Au and Pt.

\begin{tabular}{ccccc}
\hline \hline & $\epsilon$ & $E$ & $\sigma$ & $\tau$ \\
& $10^{-3} \mathrm{eV}$ & $\mathrm{GPa}$ & & $\mathrm{J} / \mathrm{m}^{2}$ \\
\hline $\mathrm{Au}(110)$ & $1.8^{a}$ & $80^{b}$ & $0.42^{b}$ & $1.0^{c}$ \\
$\mathrm{Pt}(110)$ & $2.3^{d}$ & $147^{b}$ & $0.39^{b}$ & $1.6^{c}$ \\
\hline \hline
\end{tabular}

${ }^{a}$ From Ref. [3],

${ }^{b}$ Annealed metal, from Refs. [12] and [14],

${ }^{c}$ Tangential surface stress of spherical crystallites, from Ref. [15],

${ }^{d}$ Estimated on the basis of the critical temperatures for $\mathrm{Au}(110)$ and $\mathrm{Pt}(110)$. 


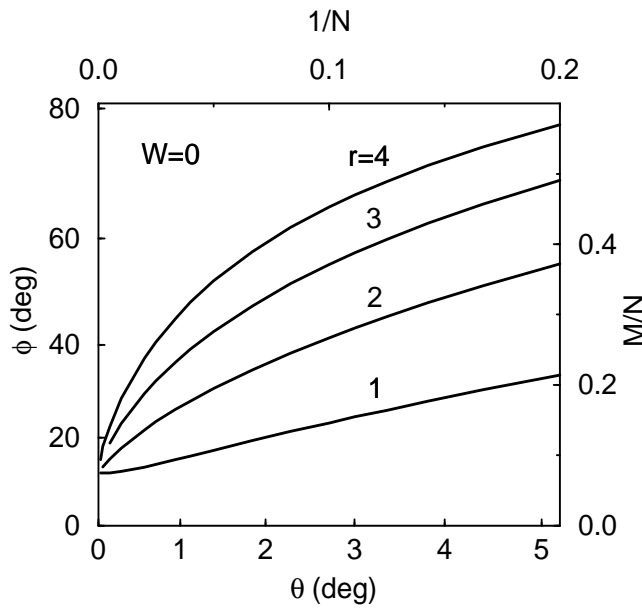

(a)

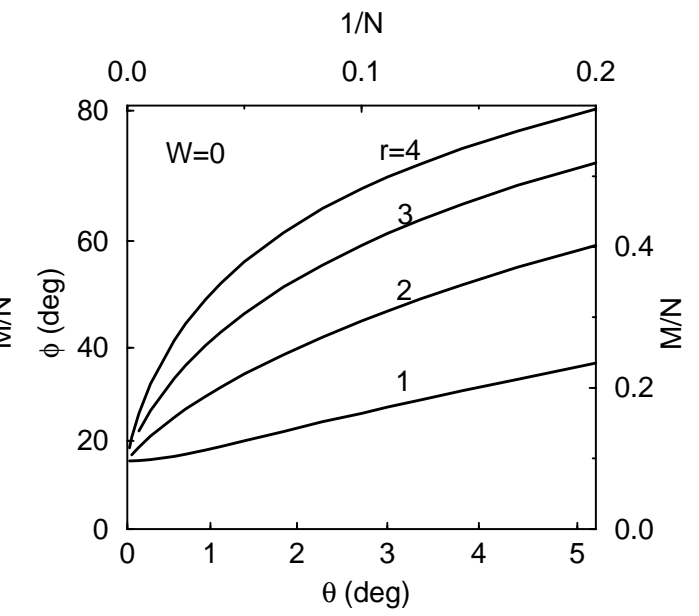

(b)

Fig. 5. Average angle between crossing steps, $\phi$, as a function of the miscut angle $\theta$ for various values of $r=\left|f_{\|} / f_{z}\right|$ and $W=0$. (a) $\mathrm{Au}(110)$, and (b) $\operatorname{Pt}(110)$.

Only in the limit as $T \rightarrow 0$ the steps become perpendicular to the miscut direction - provided they are not frozen in a finite-temperature configuration.

\section{Conclusions}

It was shown that thermally activated fluctuations of steps, constrained by the conditions of no overhangs and no crossings of the steps of the same kind, lead to the characteristic step patterns. For the missing-row reconstructed surfaces, the overhangs in the direction of missing rows are forbidden or appear with very small probability in the strong chirality regime and the overhangs in the direction perpendicular to the missing rows are virtually impossible if the anisotropy in the step energy is strong enough $(V \gg \epsilon)$. Both conditions seem to be fulfilled in the case of the reconstructed (110) noble metal surfaces, like $\mathrm{Au}(110)$ or $\mathrm{Pt}(110)$. The angle under which the opposite steps cross depends on the miscut. For small miscut angles $\left(\theta<\sim 0.2^{\circ}\right)$ or $r<\sim 1.5$, the patterns are determined by competition between the step energy and entropy. For $\theta>\sim$ $0.2^{\circ}$ and $r>\sim 2$, the competition between the elastic interaction energy and the entropy controls the step patterns. The vertex energy $W$ bears short-range elastic interactions, it is usually small and was neglected in this paper.

It would be interesting to check the temperature dependence of the step patterns experimentally. By measuring the angle between the steps, one can get insight into the elastic interaction energy between the steps. In particular, one could determine the ratio between the local stretch and local torque at the steps, $r=\left|f_{\|} / f_{z}\right|$. From the length $N^{\prime}$ of the sections with two opposite steps running next to each other, information on the vertex energy $W$ could also be 
obtained.

\section{Acknowledgements}

The author is indebted to Enrico Carlon who first pointed out the problem of step pattern formation.

\section{References}

[1] T. Gritsch, D. Coulman, R. J. Behm, and G. Ertl, Surf. Sci. 257, 297 (1991).

[2] J. K. Gimzewski, R. Berndt, and R. R. Schlittler, Phys. Rev. B 45, 6844 (1992).

[3] L. D. Roelofs, S. M. Foiles, M. S. Daw, and M. J. Baskes, Surf. Sci. 234, 63 (1990).

[4] M. E. Fisher, J. Chem. Soc., Faraday Trans. 82, 1569 (1986).

[5] J. Villain and I. Vilfan, Europhys. Lett. 12, 523 (1990).

[6] I. Vilfan and J. Villain, Surf. Sci. 257, 368 (1991).

[7] D. Cvetko et al., Surface Science 269, 68 (1992).

[8] M. A. Krzyzowski et al., Phys. Rev. B 50, 18505 (1994).

[9] V. I. Marchenko and A. Y. Parshin, Sov. Phys. JETP 52, 129 (1980).

[10] P. Nozières, in Solids Far from Equilibrium, edited by C. Godrèche (Cambridge University Press, Cambridge, 1992).

[11] J. Lapujoulade, Surf. Sci. Rep. 20, 191 (1994).

[12] J. Stewart, O. Pohland, and J. M. Gibson, Phys. Rev. B 49, 13848 (1994).

[13] S. Speller et al., Surf. Sci. Lett. 312, L748 (1994).

[14] in American Institute of Physics Handbook, edited by D. G. et al. (Mc GrawHill, New York, 1972).

[15] S. Swaminarayan, R. Najafabadi, and D. J. Srolovitz, Surf. Sci. 306, 367 (1994). 